EPJ Web of Conferences 102, 00016 (2015)

DOI: $10.1051 /$ epjconf/ 201510200016

(C) Owned by the authors, published by EDP Sciences, 2015

\title{
Modeling and interpretation of images ${ }^{\star}$
}

\author{
Michiel Min \\ ${ }^{1}$ Astronomical Institute "Anton Pannekoek", University of Amsterdam, PO Box 94249, 1090 GE, Amsterdam, \\ The Netherlands
}

\begin{abstract}
Imaging protoplanetary disks is a challenging but rewarding task. It is challenging because of the glare of the central star outshining the weak signal from the disk at shorter wavelengths and because of the limited spatial resolution at longer wavelengths. It is rewarding because it contains a wealth of information on the structure of the disks and can (directly) probe things like gaps and spiral structure. Because it is so challenging, telescopes are often pushed to their limitations to get a signal. Proper interpretation of these images therefore requires intimate knowledge of the instrumentation, the detection method, and the image processing steps. In this chapter I will give some examples and stress some issues that are important when interpreting images from protoplanetary disks.
\end{abstract}

\section{Introduction}

Imaging is becoming an increasingly important tool for studying protoplanetary disks. The reason for this is twofold. First, it is by now realized that the analysis of spatially unresolved data is highly degenerate and some information on the spatial distribution of matter is needed to lift these degeneracies. Second, with the increasing spatial resolution of large aperture telescopes and interferometric arrays it is now becoming possible to spatially resolve protoplanetary disks, also for the more regular, smaller objects. This, together with newly developed, sophisticated data analysis tools, now allows us to include imaging into our standard analysis toolkit.

Even though with modern telescopes it is often possible to resolve a protoplanetary disk, the interpretation of the image is not always straightforward. Disks are faint with respect to their central star and often tricks are needed to remove the glare from the star from the image to extract the image of the disk. These tricks are not without side-effects, and we will touch upon some of these in this chapter. I do not aim at providing a full overview of imaging techniques, coronograph masks, or types of images available. I will discuss some of the most commonly employed techniques and touch upon their strengths and quirks. I will do this through examples with no intention of being complete.

\section{Spatial resolution}

Protoplanetary disks are relatively small objects. Therefore, resolving them with a telescope is still a challenging task. When instruments and telescopes are pushed to their limits to achieve spatially resolved observations of a protoplanetary disk, it is very important to understand these limits and the

$\star 15^{\text {th }}$ Lecture from Summer School "Protoplanetary Disks: Theory and Modelling Meet Observations"

This is an Open Access article distributed under the terms of the Creative Commons Attribution License 4.0, which permits unrestricted use, distribution, and reproduction in any medium, provided the original work is properly cited. 


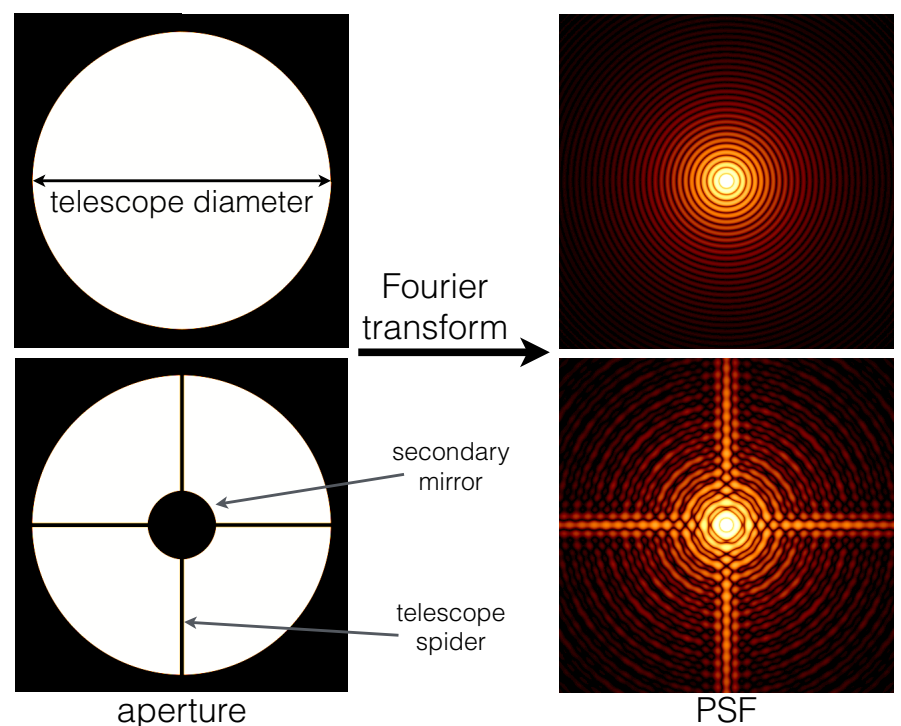

Figure 1. Schematic representation on how to obtain the PSF from the aperture of a telescope. Upper panel represents the PSF for a pure, simple aperture (also given by Eq. 1), while the lower panel represents a more complex, realistic aperture for a telescope with a secondary mirror and telescope spiders.

effects they can have on the final image. In this section we will discuss the effects of finite spatial resolution, followed by a the main methods currently employed to remove the glare from the central star from the final image.

Spatial resolution is characterized by the Point Spread Function (PSF) of the observation. The raw observed image is usually simply a convolution of the perfect image with this PSF. However, with modern tricks to improve the spatial resolution, life is not always this simple. Two notable examples are adaptive optics systems and coronographic imaging, which both have a PSF that varies strongly as a function of the location in the image.

\subsection{Telescope diffraction}

The most strict limitation on the achievable spatial resolution is given by the size of the telescope used to observe. Diffraction by the finite aperture of a telescope causes the PSF to be an airy pattern

$$
\mathrm{PSF} \propto\left(\frac{J_{1}(\pi \theta D / \lambda)}{\pi \theta D / \lambda}\right),
$$

with $\lambda$ the wavelength of radiation, $D$ the diameter of the telescope, $\theta$ the angular distance in radians, and $J_{1}$ the Bessel function of the first kind of order one. The first null of this pattern is located at

$$
\theta_{\text {null }} \approx 1.22 \frac{\lambda}{D}
$$

This is what is typically referred to as the diffraction-limited resolution of a telescope. For example, for the Hubble space telescope with a diameter $D=2.4 \mathrm{~m}$ in the V-band $(\lambda=0.55 \mu \mathrm{m})$ the diffractionlimited resolution is $2.8 \cdot 10^{-7}$ radians $=0.06$ arcsec. For mid infrared imaging with the VLT $(\lambda=$ 

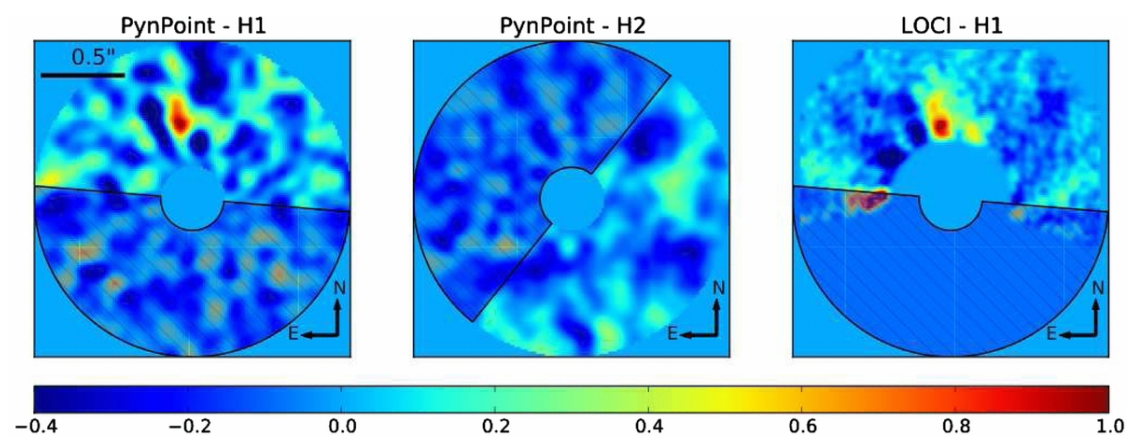

Figure 2. NACO/APP L' images of the protoplanetary disk surrounding HD 100546. An emission source can be seen in the left and right panels. The shaded area in the images indicates the region that was covered by the low sensitivity side of the apodizing phase plate. Figure taken from Quanz et al. (2013, (c) AAS, reproduced with permission).

$20 \mu \mathrm{m}, D=8.2 \mathrm{~m}$ ) the diffraction-limited resolution is only 0.6 arcsec. Note that this is only the position of the first null in the diffraction pattern, the diffraction rings can still show up in the image, which can cause difficulties even for a diffraction-limited telescope. We have to compare this with the angular size of a $100 \mathrm{AU}$ disk at 150 parsec which is only 0.67 arcsec to see that imaging of the inner regions of protoplanetary disks is currently still challenging.

The PSF given above for a perfect telescope is basically the Fourier transform of the telescope aperture. More complex PSFs, with for example spiders and a secondary mirror, can be obtained simply by taking the Fourier transform of the more complex aperture (see Fig. 1). As can be seen in Fig. 1 the effect of a finite aperture is that it removes the high spatial frequency components from the image, i.e. the small spatial scales.

\subsection{Atmospheric seeing and adaptive optics}

In most cases, when observing from the Earth, the spatial resolution is actually set by the size of the so-called seeing disk. In the optical part of the spectrum at a typical telescope site this is of the order of 0.5 to 1 arcsec. This is simply due to turbulence in the atmosphere, causing local variations of its refractive index which in turn causes the wavefront to be distorted. Thus the image of a star becomes blurred. In principle, if one has a distorted mirror with the inverse properties of the atmosphere, one could correct for this distortion and obtain a diffraction-limited image. This is exactly the idea behind adaptive optics. A deformable mirror is implemented in the light beam with the sole purpose of distorting the wavefront in exactly the opposite way as the atmosphere. To find the correct deformation of the mirror, an algorithm is implemented that tries to minimize the imaged size of a reference star in real-time. The limitations of this method are given by the number of degrees of freedom of the deformable mirror and the accuracy and speed of the algorithm doing the image minimization. The effect of this procedure is that the effects of seeing are reduced in a particular part of the image, centered on the central star. Thus the PSF is not homogeneous over the image anymore.

\section{Removing the central star}

The most important effect of finite resolution when imaging protoplanetary disks in the optical part of the spectrum is the effect of the stellar halo. The central star is so much brighter than the disk that even 


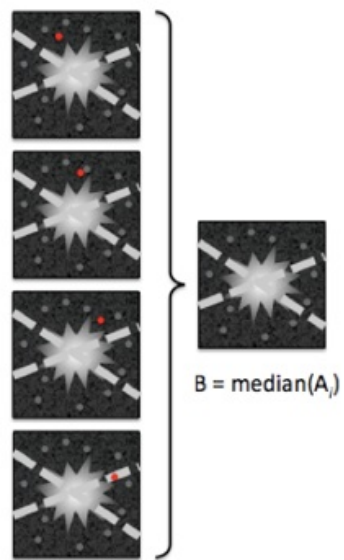

$A_{i}$

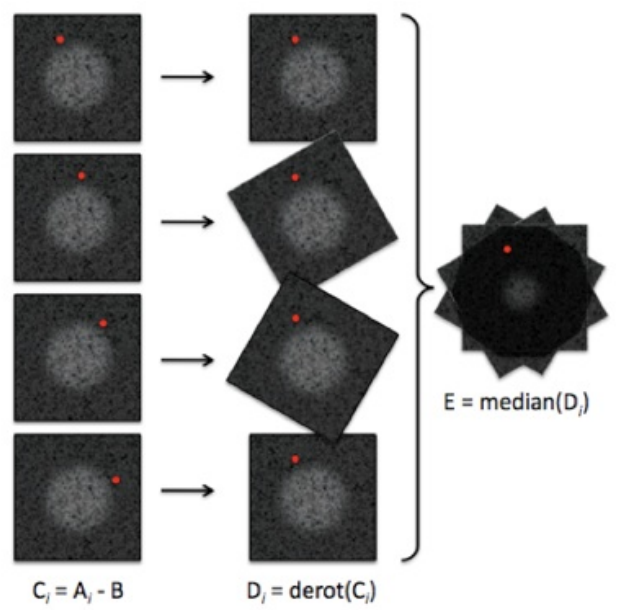

Figure 3. Illustration of the ADI method to subtract the stellar PSF from an image (credit: C. Thalmann)

the wings of the PSF are brighter, drowning the signal from the disk. There are several techniques to remove the stellar halo from the images. Below the most frequently employed methods are briefly outlined.

\subsection{Coronograph masks}

The most straightforward way to get rid of the central star is to put a small mask in the beam of the telescope which blocks its light. This does, however not automatically get rid of the diffraction effects around it. Special coronograph masks are developed to reduce the diffraction effects around the coronograph mask. For example, the apodizing phase plate coronagraph developed by Kenworthy et al. (2010) suppresses all starlight on one side of the image at the cost of a lot of starlight at the other side. This was succesfully used to image a protoplanet candidate embedded inside the protoplanetary disk surrounding HD 100546 (see also Fig. 2 from Quanz et al. 2013).

\subsection{PSF subtraction methods}

Subtracting the stellar halo from an image is probably one of the most crucial things to do to unveil the faint signal of the disk. However, since the PSF of the telescope/instrument is usually not very stable, especially in the case of atmospheric seeing, one has to measure the PSF in real-time to be able to subtract it in an accurate way. Two important techniques to do this are angular differential imaging (ADI) and polarization differential imaging (PDI). In both cases the full science image and an estimate of the PSF are in principle measured simultaneously and in the same way.

\subsubsection{Angular differential imaging (ADI)}

In ADI one uses the rotation of the sky to subtract a circular-symmetric signal from the image. The telescope and instrument settings are held constants while the object rotates on the sky, due to the rotation of the Earth, and multiple images are taken at several times. Averaging all images together, without derotating the field, one gets the average signal from all circularly-symmetric contributions in 
the image. The most important one of these is the star itself. By subtracting this average image from each frame, one is left with the contribution from non-circular-symmetric sources in the image. The method is illustrated in Fig. 3. For the signal from a point source around a star (a planet), the method is very straightforward. However, when using this signal to extract images of protoplanetary disks, one has to realize that by using ADI, one also removes all circularly-symmetric contributions of the disk itself. For example, it would not be possible to image a pole on, axisymmetric disk in this way, since the entire signal would be circularly-symmetric and thus removed.

An important improvement of classical ADI is LOCI (local optimized combination of images). For a correct estimate of the stellar PSF, it is important to have the frames over which you average to be as close as possible in time to the observed image. However, to make sure that the image of the disk is not subtracted out, it is important to have had enough image rotation. In LOCI one determines the optimal set of images to create for each frame the best reference stellar PSF to subtract.

\subsubsection{Polarimetric differential imaging (PDI)}

When light is scattered by the dust particles in the disk, it becomes polarized. With PDI one uses the assumption that the signal from the central star is unpolarized. So when we subtract the signal in two orthogonal polarization states we are in principle left with only the signal from the disk. There are two main difficulties with this method: 1) instrumental polarization, and 2) noise. The second issue, noise, comes from the fact that when using PDI on a disk that is intrinsically only $10 \%$ polarized (typically), $90 \%$ of the photons are thrown away. There is no other solution than simply integrating longer. The first issue is more challenging to tackle. Instrumental polarization is polarization introduced by the components of the telescope and instrument. Each mirror in the light-path introduces some degree of polarization, so also the initially unpolarized starlight becomes slightly polarized. One has to correct for this by either computing the exact polarization properties of the instrument and inversely apply these polarization properties to the observed images, or characterizing the polarization properties empirically. The second method is often employed in a way that it is assumed that the brightest central pixels of the image are pure starlight, and thus should be unpolarized. Subtracting the polarization signal of this central region is an effective way of removing the stellar polarization halo from the outermost regions. However, the central region of the image is usually not pure starlight. The unresolved inner edge of the disk can cause intrinsic polarization of the innermost resolution element. When the standard instrumental polarization subtraction is employed, this causes an overestimate of the instrumental polarization, which affects the derived polarized image (see Min et al. 2012, for a discussion of these effects).

PDI comes at a price, the signal observed in the image, even when instrumental polarization is perfectly corrected for, is the product of the intensity and the degree of polarization. So low flux means either low intensity, or low polarization efficiency. The most confusing, and straightforward, example of this is the variation of polarized brightness with scattering angle. This can cause the backside and front side of the disk to be significantly fainter in polarized intensity since this corresponds to forward and backward scattering which has an intrinsically low degree of polarization.

\section{Multi-wavelength imaging}

At different wavelengths we probe different aspects and regions in the disk. When interpreting images it is crucial to keep this in mind. 


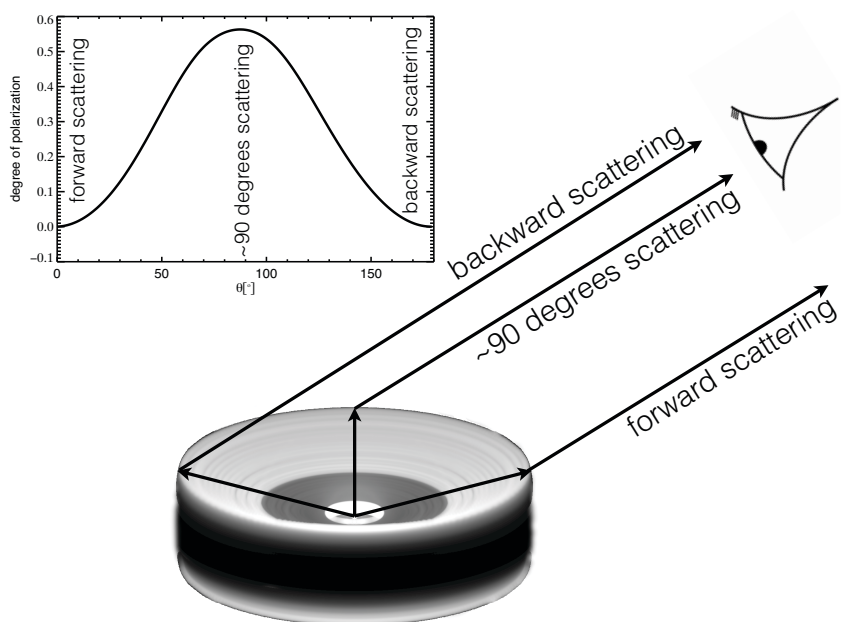

Figure 4. Illustration showing the dominating scattering angles observed from different parts of the disk. Also shown in the inset is the typical shape of a polarization phase curve. Combined the two figures give an impression of the expected degree of linear polarization as a function of the location in the image.

\subsection{Scattered light images}

At short wavelengths, the dominant radiation from the disk is in scattered light. At optical wavelengths it is pure radiation from the central star that is scattered by the grains in the surface layers of the outer disk. At near-IR wavelengths there is also a large contribution from thermal emission by the disk itself. This radiation is again scattered by the outer radii of the disk. The way in which dust grains scatter radiation depends on their properties. For a detailed description of this we refer to Min (2015). In general, the most important characteristic is anisotropic scattering. If we have large dust grains, they preferentially scatter light into the forward direction, while small particles tend to scatter light more evenly in all directions. This has an interesting effect on the brightness and color of the disk in scattered light, as is extensively discussed in Mulders et al. (2013). The main effect is that for highly forward-scattering particles, the scattered light disappears into the disk, while for more isotropically scattering particles the light is scattered towards the observer (see also Fig. 5). Thus, for large grains the disk becomes faint. Interestingly, large implies large with respect to the wavelength of observation. Thus the disks also become fainter at shorter wavelengths, which might explain the red scattering color observed in, for example, the disk around HD 100546 (Mulders et al. 2013) and HD 163296 (Wisniewski et al. 2008).

The scattered light we observe from the outer region of the disk actually originates from the central star (or the inner edge of the disk). Thus it already traveled through the inner regions of the disk as well. The brightness observed in scattered light can therefore be heavily affected by shadowing by the innermost regions. This is, for example, interpreted to be the cause of the varying brightness of the outer disk around HD 163296 in scattered light (Wisniewski et al. 2008). In addition, the scattering happens on the uppermost layers of the outer disk. Thus the exact structure of the outer disk, the flaring angle, the degree of grain settling, has a very large impact on the brightness in scattered light. 


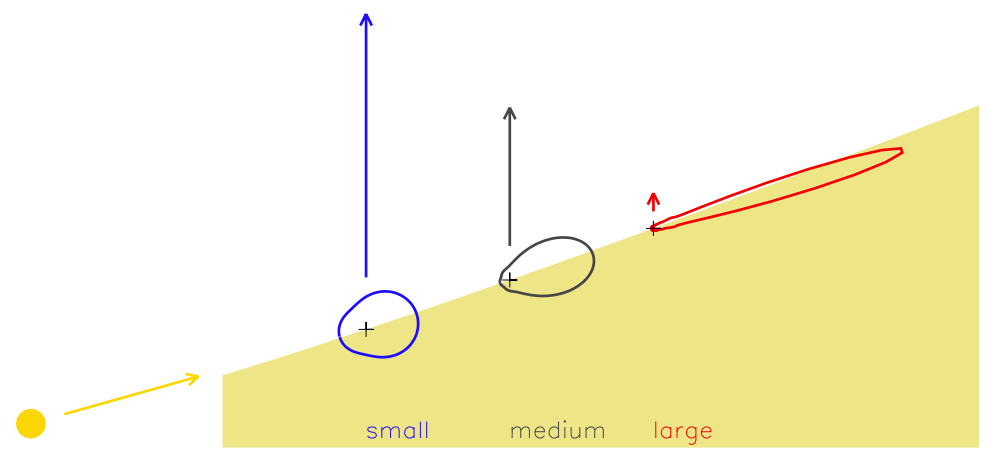

Figure 5. Illustration of how anisotropic scattering influences the intensity of the scattered light observed. Figure taken from Mulders et al. (2013, reproduced with permission (C) ESO)

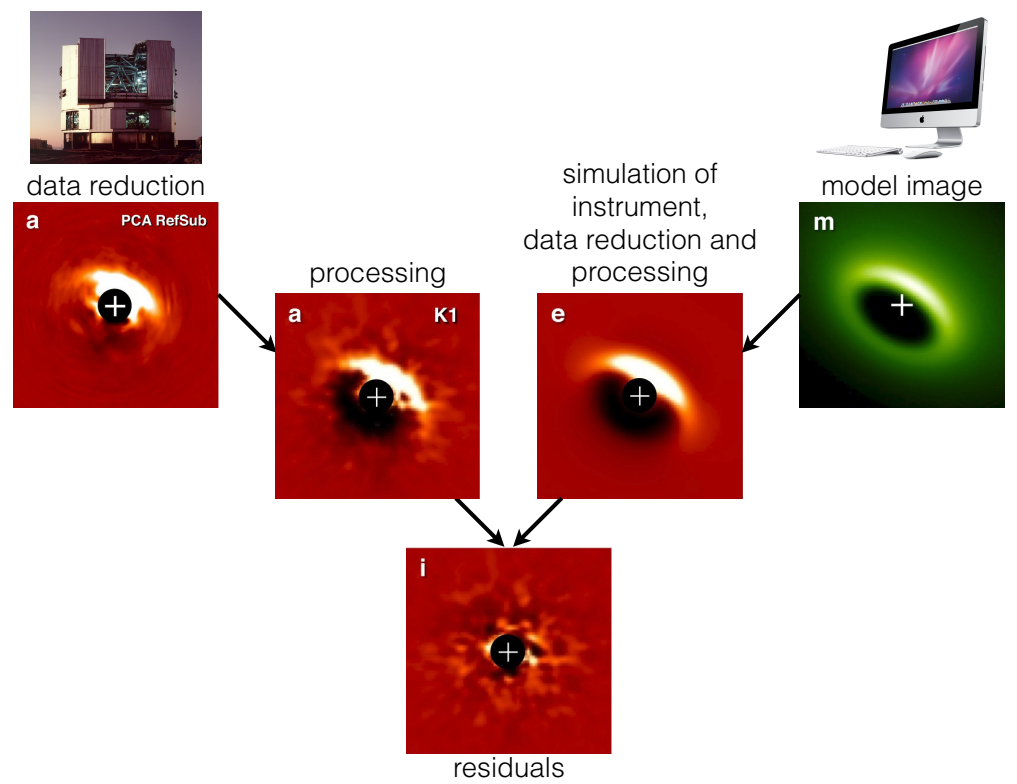

Figure 6. The chain of processes from observation to final science image (left branch) and from model setup to simulated observation (right branch). The example shown is the near-infrared scattered light image of the disk surrounding LkCa15 processed using ADI/LOCI (Thalmann et al. 2014).

\subsection{Infrared imaging}

In the infrared part of the spectrum we observe the thermal emission from the disk itself, and the central star becomes much less important. This implies that removal of the PSF is of lesser importance. However, at near infrared wavelengths, the central region of the disk might still outshine structures in the outer disk. In addition, the spatial resolution when observing with the same aperture but at longer wavelengths becomes increasingly poor (see also the mid-infrared imaging study by Doucet et al. 2006). An interesting study using mid-infrared imaging of protoplanetary disks was performed by Maaskant et al. (2013). Here it is concluded that if a disk has a gap, the wall of the gap creates a 


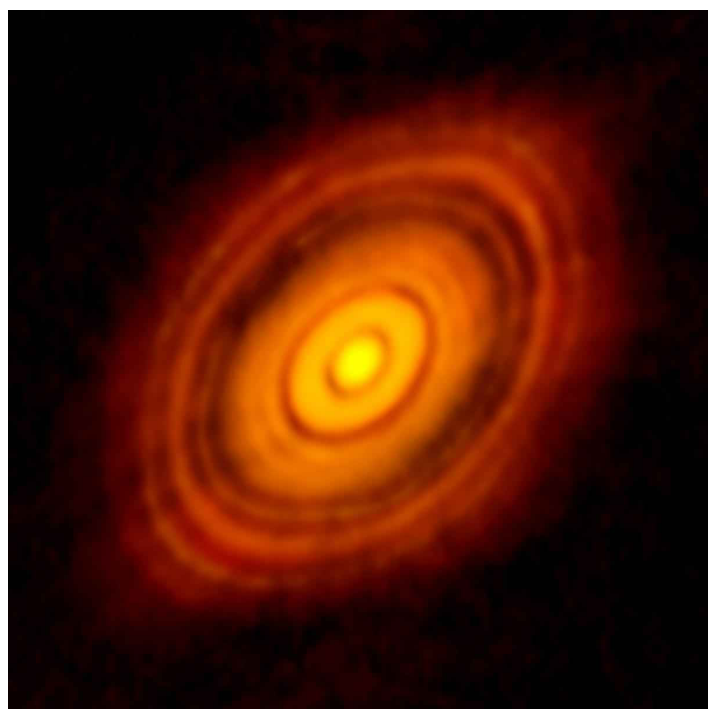

Figure 7. Astonishing ALMA image of the disk around HL Tau. A large number of gaps can be seen, the origin of which is still heavily debated. Credit: ALMA (NRAO/ESO/NAOJ); C. Brogan, B. Saxton (NRAO/AUI/NSF)

large surface area, radiating very strongly at mid infrared wavelengths. This can generate a spatially resolvable signal that can be used to estimate the size of the gap while a continuous, gapless disk would not be resolved at mid-IR wavelengths as the dominating emission then originates further in.

The mid infrared wavelength range also contains many solid state and PAH (Polycyclic Aromatic Hydrocarbon) features. Differential imaging at different wavelengths can therefore be used to generate composition maps. Up to now, this has mainly been possible using the PAH features. For example, in Doucet et al. (2007) the flaring structure of the disk surrounding HD 97048 was imaged in the $11.3 \mu \mathrm{m}$ PAH filter. Interestingly, compositional analysis using imaging can also sometimes be done using scattered light. Honda et al. (2009) used scattered light around $3 \mu \mathrm{m}$ to identify the $3 \mu \mathrm{m}$ ice feature in the outer disk of HD 142527.

\subsection{Millimeter imaging}

At millimeter wavelengths the disk becomes optically thin and we can probe the mass of the disk. At these wavelengths all dust grains smaller than $\sim 1 \mathrm{~mm}$ contribute equally by mass to the emission and the temperature dependence also is only linear. Therefore, millimeter imaging is the ideal tool to probe where most of the mass in dust grains in a disk resides. Because of the strong dependence of spatial resolution with wavelength, millimeter imaging with a single dish telescope is not useful. With the arrival of ALMA, we can now make images of unprecedented spatial resolution at millimeter wavelength (see Fig. 7 for an amazing example). Combined continuum and line imaging in the millimeter provides important information on the spatial distribution and separation of gas and dust in the disk (see Ilee \& Greaves 2015).

\section{Comparing model and observation}

Image processing, especially for scattered light images, removing the central PSF, has become a crucial tool in extracting images from observations. These image processing algorithms leave very strong 

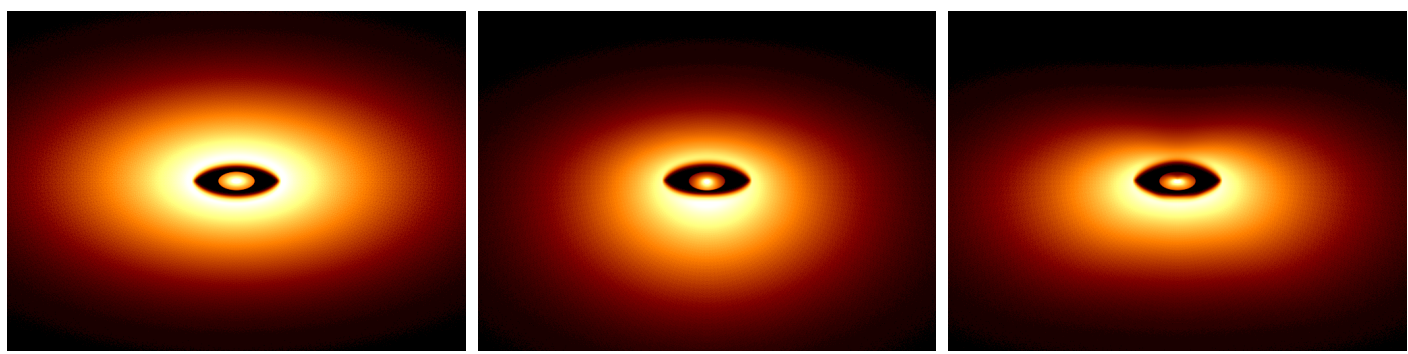

Figure 8. Model images of a typical transitional disk setup at optical wavelengths. Left image shows the image computed assuming isotropic scattering, center image using full anisotropic scattering. Rightmost image shows the polarized intensity. The backward tilted side of the disk is up in the image. The disk itself is perfectly axisymmetric and tilted by 60 degrees with respect to face-on.

traces and artifacts in the final images. It is therefore not always straightforward to interpret a final image. The way to solve this problem is to generate model images and simulate the entire process: observation by the telescope, alteration by the instrument, and cleaning by the image processing tools to generate a simulated observation from the model. A simulated observation can then be directly compared to the real observation. A good example, using ADI/LOCI on the disk surrounding LkCa15, is the study by Thalmann et al. (2014) obtaining the geometrical parameters of the transition disk (see also Fig. 6).

\section{Interpreting observed structures}

\subsection{Asymmetries}

The first analysis of an image, before any modeling, is done by eye. When doing this it is good to realize that humans are not trained to correctly interpret something as complex as a protoplanetary disk over the entire wavelength spectrum. Effects of extreme optical depths, highly anisotropic scattering, and polarization degree can cause structures that might be interpreted as disk structure or density fluctuations. For example, the previously already discussed image of LkCa15 (see Fig. 6) was first intuitively interpreted as seeing the illuminated backside of the inner edge of the outer disk. However, more detailed modeling showed that this interpretation cannot reproduce the highly asymmetric structure seen, and the real effect is strong forward scattering off the front side of the disk. Fig. 8 shows computations of a typical transitional disk setup to illustrate the asymmetries that can be created by anisotropic scattering or polarization (center and right panels). Also note that, as discussed before, the disk is much fainter overall when we consider anisotropic scattering (compare left and middle panels).

With ALMA, highly asymmetric structures have been observed at millimeter wavelengths in the continuum (see e.g. Casassus et al. 2013). Since the disk is optically thin at millimeter wavelengths and the radiation is from thermal emission (which is isotropic), at these wavelengths the only explanation is a true asymmetry of the disk. It is interpreted as being caused by Rosby wave instabilities that lead to the creation of a vortex, providing a long-lived density enhancement into which the large particles are drifting azimuthally. This has triggered important discussions on the possibilities of so-called dust-traps (Birnstiel et al. 2013; Klahr \& Henning 1997). 

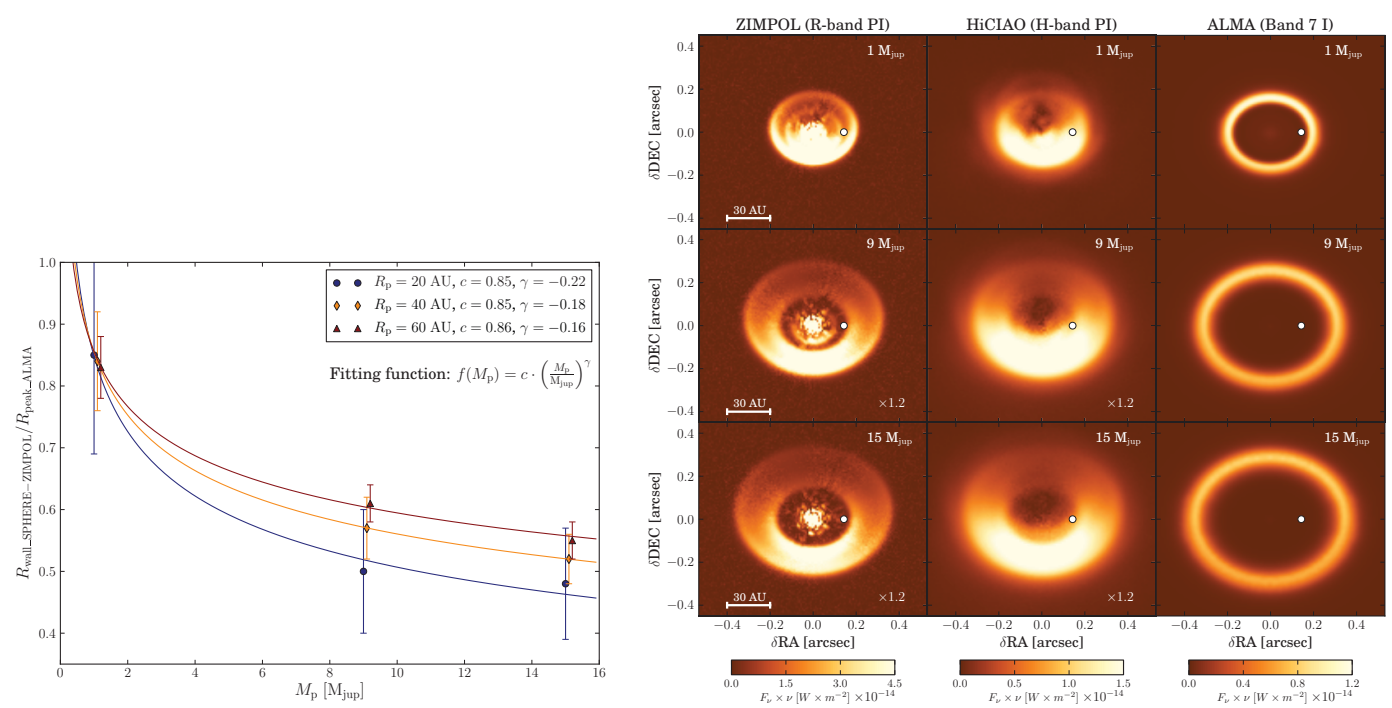

Figure 9. Simulated observations of a disk with a planet carving a gap. The gravitational push on the gas is a strong function of the planet mass. This creates differences in the radial drift behavior of the grains and the apparent size of the carved out gap at different wavelengths that probe different grain sizes. The right figure provides an empirical way to derive the mass of the unseen planet by measuring the size of the gap at different wavelengths. Figures taken from (de Juan Ovelar et al. 2013, reproduced with permission (c) ESO).

\subsection{Gaps in transitional disks}

Polarimetric images of protoplanetary disks taken in the framework of the SEEDS survey revealed a very interesting aspect. It appeared that in a number of objects, where a gap was detected using millimeter interferometry, this gap was not detected in scattered light in the optical part of the spectrum (Dong et al. 2012). Currently, the most widely accepted explanation is the presence of a large planet in the disk. A sufficiently massive planet creates a gap in the disk gas by adding angular momentum to the gas outside and removing angular momentum from gas on the inside. This process creates a pressure maximum in the outer disk, significantly removed even from the outer gap edge. Large grains, which contain most of the disk mass, drift toward the pressure maximum and are collected their, causing the characteristic ring structure seen in submm interferometric observations. Small grains are coupled strongly to the gas, and their distribution shows the inner edge of the outer disk rather than the pressure maximum. This causes the scattered light images to have a significantly smaller gap size (scattered light is caused by the small grains in the surface layer) than the millimeter images, which probe the mass of the disk (and thus the large grains). This was studied theoretically by de Juan Ovelar et al. (2013) using hydrodynamical simulations and radiative transfer to predict gap sizes as a function of wavelength for different planetary masses. The result of that study is that we might be able to use the difference between the millimeter and optical gap size to constrain the mass of the planet shaping the gap (see also Fig. 9).

\subsection{Spiral waves}

Spiral structures have been reported in a significant fraction of resolved protoplanetary disks. Two mechanisms have been proposed to create spiral structure in disks, 1) a massive planet, 2) a gravita- 
Summer School "Protoplanetary Disks: Theory and Modeling Meet Observations"

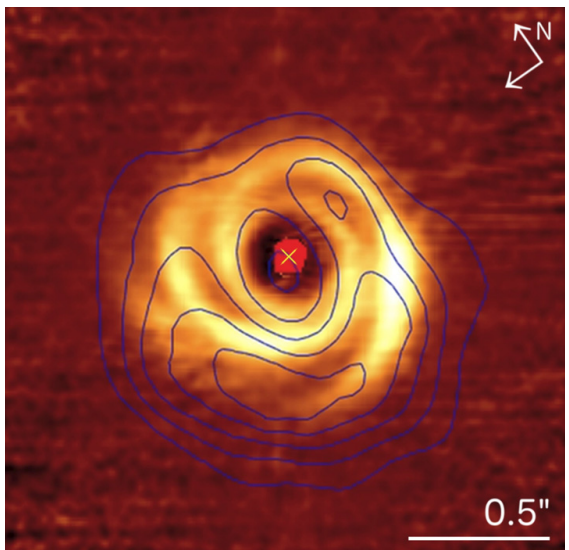

Figure 10. Spiral structure observed in the disk around SAO206462. Image taken from Garufi et al. (2013, reproduced with permission (C) ESO).

tional instability in the disk. The amplitude of the density fluctuations created by these two mechanisms is quite different. A massive planet in the disk will cause spiral density waves with only a small amplitude, and it is still an open question if this will be sufficient to create the large contrast seen in some scattered light images (see e.g. Fig. 10 taken from Garufi et al. 2013). A discussion on the detectability of spiral waves is presented in Juhasz et al. (2014). On the other hand, spiral waves caused by gravitational instabilities create a much larger density contrast (up to an order of magnitude, see Dipierro et al. 2014). These density fluctuations would be detectable also with ALMA in the surface brightness at millimeter wavelengths. Indeed Pérez et al. (2014) report tentatively that spiral structure can be seen when carefully analyzing the ALMA images of SAO 206462. At this moment, the question wether the detected spiral structures are caused by planets or gravitational instabilities remains open.

\section{Summary}

Images are one of the most instructive observables we can get. However, they have to be handled with care. Human intuition is not built to interpret images of protoplanetary disks, and we should rely on firmly established trends through detailed modeling. When using telescopes at the edge of their abilities, modern data reduction and post processing techniques can perform very well to extract a wealth of information. This comes at a price, which is to make the images sometimes even harder to interpret. The solution to this issue is forward modeling, where the model image is put through a simulation chain including atmosphere, telescope, instrument, data reduction and post processing to be able to directly compare to observations.

Asymmetries in disks can be caused by optical depth, anisotropic scattering, polarization effects (in the case of PDI), or intrinsic asymmetries in the disk. These can be density fluctuations, but also fluctuations in grain size or composition, or fluctuations of the local turbulence, which can cause the vertical extend of the disk to vary. Careful analysis of these possibilities should be done to extract the optimal amount of information.

We are currently at a stage where spatially resolving the regions in protoplanetary disks where planets form is becoming standard business. These images contain precious information on the formation of planets in disks, and we have to pull out all we can to extract this information. Many 
questions and degeneracies we have from modeling spatially unresolved data will be answered and removed by using these images if we allow ourselves to take the time to robustly interpret what we see in the context of physical disk models.

Acknowledgements The research leading to these results has received funding from the European Union Seventh Framework Programme FP7-2011 under grant agreement no 284405.

\section{References}

Birnstiel, T., Dullemond, C. P., \& Pinilla, P. 2013, A\&A, 550, L8

Casassus, S., van der Plas, G., M, S. P., et al. 2013, Nature, 493, 191

de Juan Ovelar, M., Min, M., Dominik, C., et al. 2013, A\&A, 560, A111

Dipierro, G., Lodato, G., Testi, L., \& de Gregorio Monsalvo, I. 2014, MNRAS, 444, 1919

Dong, R., Rafikov, R., Zhu, Z., et al. 2012, ApJ, 750, 161

Doucet, C., Habart, E., Pantin, E., et al. 2007, A\&A, 470, 625

Doucet, C., Pantin, E., Lagage, P. O., \& Dullemond, C. P. 2006, A\&A, 460, 117

Garufi, A., Quanz, S. P., Avenhaus, H., et al. 2013, A\&A, 560, A105

Honda, M., Inoue, A. K., Fukagawa, M., et al. 2009, ApJ, 690, L110

Ilee, J. \& Greaves, J. 2015, in EPJ Web of Conferences, Vol. 102, Summer School on Protoplanetary Disks: Theory and Modeling Meet Observations, ed. I. Kamp, P. Woitke, \& J. D. Ilee

Juhasz, A., Benisty, M., Pohl, A., et al. 2014, ArXiv e-prints

Kenworthy, M. A., Quanz, S. P., Meyer, M. R., et al. 2010, in Society of Photo-Optical Instrumentation Engineers (SPIE) Conference Series, Vol. 7735, Society of Photo-Optical Instrumentation Engineers (SPIE) Conference Series

Klahr, H. H. \& Henning, T. 1997, Icarus, 128, 213

Maaskant, K. M., Honda, M., Waters, L. B. F. M., et al. 2013, A\&A, 555, A64

Min, M. 2015, in EPJ Web of Conferences, Vol. 102, Summer School on Protoplanetary Disks: Theory and Modeling Meet Observations, ed. I. Kamp, P. Woitke, \& J. D. Ilee

Min, M., Canovas, H., Mulders, G. D., \& Keller, C. U. 2012, A\&A, 537, A75

Mulders, G. D., Min, M., Dominik, C., Debes, J. H., \& Schneider, G. 2013, A\&A, 549, A112

Pérez, L. M., Isella, A., Carpenter, J. M., \& Chandler, C. J. 2014, ApJ, 783, L13

Quanz, S. P., Amara, A., Meyer, M. R., et al. 2013, ApJ, 766, L1

Thalmann, C., Mulders, G. D., Hodapp, K., et al. 2014, A\&A, 566, A51

Wisniewski, J. P., Clampin, M., Grady, C. A., et al. 2008, ApJ, 682, 548 